University of Nebraska - Lincoln

DigitalCommons@University of Nebraska - Lincoln

February 1989

\title{
A complete set of elastic constants of crystalline anthracene by Brillouin scattering
}

\author{
R.C. Dye \\ University of Nebraska - Lincoln \\ Craig J. Eckhardt \\ University of Nebraska - Lincoln, ceckhardt1@unl.edu
}

Follow this and additional works at: https://digitalcommons.unl.edu/chemistryeckhardt

Part of the Chemistry Commons

Dye, R.C. and Eckhardt, Craig J., "A complete set of elastic constants of crystalline anthracene by Brillouin scattering" (1989). Craig J. Eckhardt Publications. 23.

https://digitalcommons.unl.edu/chemistryeckhardt/23

This Article is brought to you for free and open access by the Published Research - Department of Chemistry at DigitalCommons@University of Nebraska - Lincoln. It has been accepted for inclusion in Craig J. Eckhardt Publications by an authorized administrator of DigitalCommons@University of Nebraska - Lincoln. 


\title{
A complete set of elastic constants of crystalline anthracene by Brillouin scattering
}

\author{
R. C. Dye a) and C. J. Eckhardt ${ }^{\text {b) }}$ \\ Department of Chemistry, University of Nebraska-Lincoln, Lincoln, Nebraska 68588-0304
}

(Received 26 August 1988; accepted 1 November 1988)

\begin{abstract}
All 13 elastic constants of a vapor grown, uncut, anthracene single crystal were determined from acoustic phonon velocities obtained by the Brillouin scattering method. The phonon velocities are plotted for three crystallographic planes containing the crystal axis. The relationships between phonon velocities and lattice dynamics are discussed. A minimizing procedure is introduced for converting phonon velocities of low symmetry systems into elasticity coefficients. This is shown to have several advantages over previous methods used. The results are compared with those obtained by previous studies of anthracene elastic constants.
\end{abstract}

\section{INTRODUCTION}

Many properties of organic crystals are primarily determined by the molecular packing in the crystal and intermolecular forces. This is certainly the case for thermal and mechanical properties and partly true for electrical ones. To exploit the properties of a material to its fullest, a complete understanding of the lattice dynamics is desirable. Also, much of the information acquired from studying organic molecular systems can be extended to macromolecular systems. For these reasons the study of molecular packing and intermolecular forces has become an important area of research.

For many years there has been considerable theoretical and experimental interest in the lattice properties of crystalline anthracene. In this hydrocarbon, the lattice cohesion is mainly due to van der Waals interactions. This provides a large separation in energy between the internal molecular modes and the external lattice modes. Using this fact, theoretical calculations can be simplified by treating the molecules as rigid. However, while the theoretical calculations predict lattice energies rather well, agreement with experimental dynamical properties such as elasticity is not as consistent. In the case of anthracene, a major problem for theoretical calculations is the lack of consistent experimental results with which to compare values. With more reliable experimental results a greater understanding of molecular crystals can be obtained.

A major goal of this study is to clarify the effects packing and molecular structure have on phonon propagation in the solid state of anthracene. A sensitive tool in studying phonon propagation is the elasticity tensor since the elements of this tensor, the elastic constants, are related to the phonon velocities. Although other studies of phonons in anthracene have been done, ${ }^{1-6}$ the values reported for the elastic constants vary a great deal. To develop an understanding of the interactions in molecular crystals, it is important to obtain consis-

\footnotetext{
a) Work completed in partial fulfillment of requirements for the Ph.D. at the University of Nebraska-Lincoln.

b) Author to whom correspondence should be addressed.
}

tent experimental values in simple hydrocarbons such as anthracene. Once consistent values are acquired, improvement on theoretical models can be made.

The theoretical ground work for anthracene was broken by Pawley. ${ }^{1}$ In that work a lattice dynamical model is presented using intermolecular forces of the "6-exp" interatomic type. ${ }^{2}$ At that time no experimental phonon velocities or elastic constant values were available to test the accuracy of this atom-atom potential model for anthracene. Later, Afanaseva et $a l^{3}$ compared the results of this model with their phonon velocities and elastic constants which were obtained from the ultrasonic pulse method. The calculated values showed generally good agreement with experiment. However, these investigators realized there should be improvement between these values. Danno and Inokuchi ${ }^{4}$ also used the ultrasonic pulse method to acquire a set of elastic constants, but little refinement in agreement with the overall theoretical values was observed. Another ultrasonic study was performed by Huntington et al..$^{5}$ For that study many of the elastic constants agree rather well with Pawley's theoretical work, but 3 out of the 13 possible elastic constants were approximated and there was some difficulty in establishing the signs of two other values. Elnahwy et al. ${ }^{6} \mathrm{em}-$ ployed neutron diffraction techniques to determine the linear compressibilities of anthracene. These values were then used to obtain 10 out of the 13 possible elastic constants.

There are several problems associated with the previous methods of obtaining phonon velocities and elastic constants. All four of the previous experimental studies used Bridgeman grown samples. This type of growth can cause internal strains and high defect concentrations in molecular crystals. The previous studies also required cutting the samples which can generate inhomogeneities in the material.

Obtaining the elastic constants from the phonon velocities directly is not trivial and often requires solving simultaneous cubic or quadratic equations. The general calculative methods used in obtaining the elastic constants can induce inconsistencies in the results, since judicious choices must be made for intermediate quadratic or cubic solutions. The current study provides an added method to clarify the discrepancies in these elastic constant values. 
In this paper, a brief theory section on Brillouin scattering is presented. Subsequently, a method for converting sound velocities into elasticity coefficients is demonstrated. This is shown to have several advantages over previous methods used. Along with elastic constant values, the molecular structure and packing effects on phonon velocities in anthracene are treated in a following section. Finally, a discussion of the present results and a comparison with other work is given.

\section{THEORY}

In Brillouin spectroscopy the inelastic scattering of photons from acoustic lattice vibrations is observed. ${ }^{7}$ This process can be explained classically as Bragg diffraction from the moving grating formed by the vibrating periodic lattice. The vibrations associated with translations of mass in the unit cell, acoustic phonons, move through the lattice at the speed of sound. This phonon velocity depends on how effectively the mass in the unit cell can be moved. This is directly related to the force constants holding the mass in place.

Scattering in an anisotropic medium like anthracene admits fewer assumptions than generally presented for higher symmetry lattices. Let $\mathbf{k}_{s}, \mathbf{k}_{i}$, and $\mathbf{q}$ denote the wave vectors of the scattered, incident, and acoustic wave, respectively. Conservation of momentum requires

$$
\mathbf{k}_{s}=\mathbf{k}_{i} \pm \mathbf{q}
$$

and because of conservation of energy:

$$
w_{s}=w_{i} \pm w_{a},
$$

where $w_{s} w_{i}$ and $w_{a}$ are the energy of the scattered, incident, and acoustic wave, respectively. Taking these two equations and using the law of cosines the following obtains

$$
d v=v_{0}(v / c)\left(n_{i}^{2}+n_{s}^{2}-2 n_{i} n_{s} \cos \theta\right)^{1 / 2},
$$

the Brillouin shift is $d v$ and $v_{0}$ is the frequency of the laser light assuming the incident and scattering light are the same in frequency. The velocity of the phonon is $v$, and $c$ is the velocity of light in vacuum. The indices of refraction of the incident and scattered light are $n_{i}$ and $n_{s}$, respectively, and $\theta$ is the scattering angle.

\section{EXPERIMENTAL}

\section{Sample preparation}

Anthracene packs in a monoclinic unit cell with a space group symmetry of $P 2_{1} / a$. The unit cell contains two translationally inequivalent molecules. Lattice constants at $290 \mathrm{~K}$ are $a=8.562 \AA, b=6.038 \AA, c=11.184 \AA$, and $\beta=124.7^{\circ} .^{8}$ The plate sublimed crystals of anthracene were provided by Professor Norbert Karl. The tabular crystal used was $3 \times 5 \times 9 \mathrm{~mm}$. The interior of the crystal was of superior optical quality, however, some surfaces did have sporadic defects. Identification of the crystal faces was achieved by optical goniometry and morphological examination. Well developed $(001),(\overline{1} 01),(\overline{2} 01),(\overline{1} 11)$, and (110) faces were commonly observed. The angles between the face normals are important in determining the scattering angle and position of the crystal.

\section{Instrumentation}

The Brillouin scattering instrumentation (Fig. 1) differs somewhat from that normally used and will be treated in greater detail elsewhere. The source consists of a lightstabilized, single-moded argon ion laser. Light is passed through a half-wave plate mounted on a rotating stage which permits the incident beam to have the proper polarization and direction. The sample is placed on a goniometer attached to another rotating stage allowing appropriate crystal orientation. Scattered light is analyzed with a rotating polaroid film. This unique four-circle system provides the versatility needed to obtain q-vector directions which are inaccessible by conventional means without cutting the crystal. After passing through an iris, the light is gathered by a lens. A collimator with a $0.2 \mathrm{~mm}$ pinhole transfers the light to a multipass piezoelectrically driven Burleigh Fabry-Perot interferometer. Stabilization of the interferometer is achieved by use of a thermal box, a vibration isolation table, and an active stabilization system. This system can maintain a finesse greater than $\mathbf{7 0}$ for an extended period of time. Conventional photon counting techniques and instrumentation are employed for the detection of the scattered light. For rapid data collection and display an IBM 9000 microcomputer is used.

\section{Method of measurement}

Three scattering schemes were employed: $90^{\circ}$, near $180^{\circ}$, and with incident and scattered beams normal to nonnormal, natural faces. Back reflection of the laser beam from the incident crystal surface provided an alignment point for the scattering setup. Since this reflection is collinear with the vector normal to the face, the stage angle and crystal orientation can then be set according to the predetermined scattering scheme. Using this alignment technique an accuracy of better than $0.5^{\circ}$ could be obtained in the scattering angle.

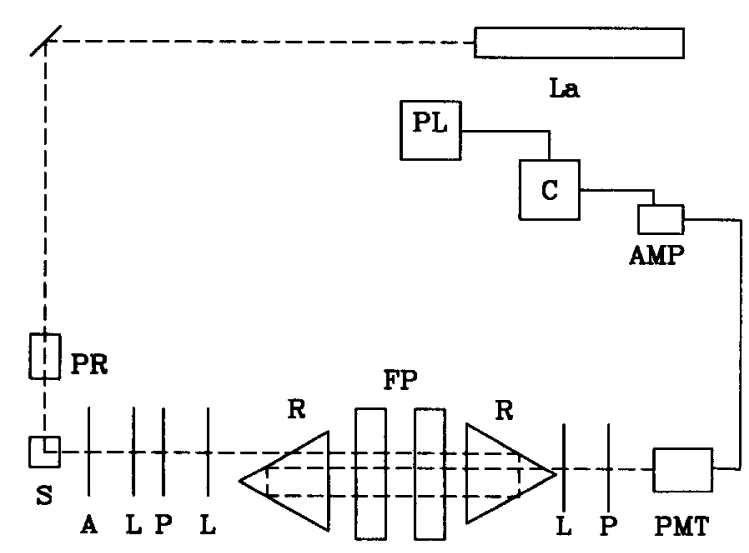

FIG. 1. Brillouin scattering setup. La: laser; PR: polarizing rotator; S: sample; A: analyzer; L: lens; P: pinhole; R: corner cube retroreflectors; FP: Fabry-Perot interferometer; PMT: photomultiplier tube; AMP: amplifier discriminator; C: multichannel analyzer; PL: plotter. 
In general, light normal to a crystal surface can propagate in the bulk in two major directions. Therefore, if the incident beam is polarized and the scattered beam is analyzed, there exist four possible scattering arrangements. In knowing angles between normals and the axes of the optical indicatrix, the beam directions and refractive indices were obtained.

To locate superposed peaks, measurements were made with at least two free spectral ranges (FSR). The FSR extended from 0.898 to $1.420 \mathrm{~cm}^{-1}$. Crystalline quartz as well as liquid benzene were employed in determining the FSR, since there is substantial agreement on their energy shifts. ${ }^{10-12}$ The Brillouin lines are, in general, narrow and symmetric. Therefore, the Brillouin shift was determined from the position of the peak center. ${ }^{13}$ In polarized scattering studies depolarization of the beam is often a problem. ${ }^{14}$ Some depolarization of the incident and scattered beam was observed in this study. Causes for the depolarization include surface roughness and internal defects. For instance, the same energy shift in the VV scattering plane might be observed in the VH arrangement. Here $\mathrm{V}$ designates light polarization perpendicular to and $\mathrm{H}$ that parallel to the scattering plane. The first position designates the polarization of the incident beam and the second position that of the scattering ray. In this case the peak in the VH arrangement would be disregarded as breakthrough scattering.

A typical $90^{\circ}$ Brillouin scattering spectrum is shown in Fig. 2. The spectrum is the result of 50 summed scans, with no smoothing.

\section{CALCULATIONS}

The elastic constant tensor for a monoclinic crystal consists of 13 independent coefficients, where $c_{i j}=c_{j i}$. The elements are shown in Eq. (4) expressed in the usual Voigt notation $^{15}$

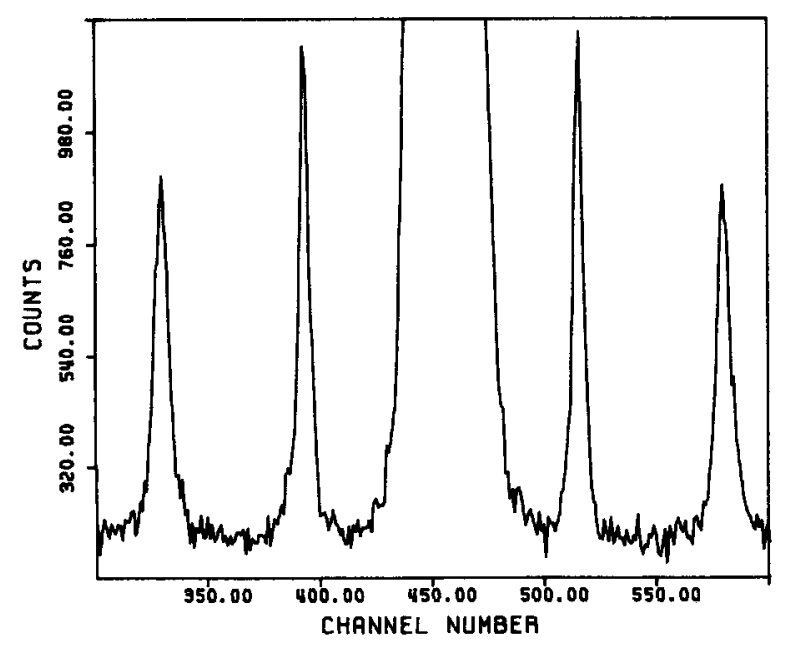

FIG. 2. Anthracene Brillouin spectrum with beam incident on the $\overline{2} 01$ face and light collected from 001 face, FSR $=1.420 \mathrm{~cm}^{-1}$.

$$
C=\left[\begin{array}{cccccc}
c_{11} & c_{12} & c_{13} & 0 & c_{15} & 0 \\
c_{21} & c_{22} & c_{23} & 0 & c_{25} & 0 \\
c_{31} & c_{32} & c_{33} & 0 & c_{35} & 0 \\
0 & 0 & 0 & c_{44} & 0 & c_{46} \\
c_{51} & c_{52} & c_{53} & 0 & c_{55} & 0 \\
0 & 0 & 0 & c_{64} & 0 & c_{66}
\end{array}\right]
$$

The computation of the elastic constants requires solution of the Christoffel equation for different directions in the lattice $^{15}$ :

$$
\left|\Gamma_{i j}(\mathbf{q})-\rho v^{2} \mathbf{1}\right|=0
$$

in which $\rho$ is the crystal density, $v$ is the elastic wave velocity, and $\Gamma_{i j}(\mathbf{q})=c_{i k j l} q_{k} q_{l}$. The elastic constants $c_{i k j l}$ are written in full index notation; also, $q_{k}$ and $q_{l}$ are the direction cosines for the angle of the wave vector to the orthogonal reference axes $k$ and $l$. When the elastic constants are known, the solution of the Christoffel equation is trivial. However, the elastic wave velocities are the values determined by experiment. Generally, the elastic constants are solved by selecting special wave vector directions that have simple elastic constant dependence. For instance, the (010) longitudinal wave is determined only by the $C_{22}$ element. This approach, however, is very limited since only a few such propagation directions with single elastic constant dependence are possible.

Cutting the crystal is usually resorted to in order to obtain special propagation directions that do not form naturally. This can severely degrade the surface as well as generate undetected cracks and other defects in the bulk. Cutting requires large crystals which are also more prone to high defect concentration. The defects may seriously affect the Brillouin line shapes and intensities and increase the Rayleigh wings.

By solving for the special directions first, the errors in the wave velocities accumulate and propagate so that the last determined tensor elements are associated with large uncertainties. Ambiguities also arise when solving the quadratic or cubic equations which express the velocity dependence on the elastic constants.

In this study a different approach is taken in solving for the elastic constants. The method was first applied to cubic crystals ${ }^{16}$ and has recently been extended to lower symmetry crystals to obtain elastic constants. ${ }^{17}$

The procedure based on a linear least-squares approach consists of an iterative optimization algorithm which starts with an arbitrary set of elastic constants. Briefly, each iteration solves the Christoffel determinants for their eigenvalues $\rho v_{\text {calc }}^{2}$. Next, the program calculates an error vector $\mathbf{e}$ between the calculated eigenvalues and the observed eigenvalues $\rho v_{\text {obs }}^{2}$ which is obtained from experimental velocities. The square of the error vector,

$$
\mathbf{e}^{T} \mathbf{e}=\Sigma\left|\rho v_{\text {obs }(\mathbf{q}, j)}^{2}-\rho v_{\text {calc }(\mathbf{q}, j, C)}^{2}\right|^{2}
$$

is minimized by systematically varying the elastic constants. The iterations continue until there is no further improvement in the values. Appropriate weights are added to correspond to the uncertainty in the observed measurements. Increasing the number of observed phonon directions improves the results rather than causing greater ambiguities. This method provides a better overall scheme for the determination of the complete set of elastic constants. Further, it 
TABLE 1. Anthracene elastic constants.

\begin{tabular}{|c|c|c|c|c|c|c|c|}
\hline & \multicolumn{7}{|c|}{$\left(10^{9} \mathrm{dyn} / \mathrm{cm}^{2}\right)$} \\
\hline & $1^{\mathrm{a}}$ & $2^{\mathbf{b}}$ & $3^{\mathrm{c}}$ & $4^{d}$ & $5^{e}$ & $6^{\mathrm{f}}$ & $7^{8}$ \\
\hline$C 11$ & 85.2 & 72.0 & 89.2 & 90.0 & 79.0 & 91.7 & 0.7 \\
\hline$C 12$ & 67.2 & 40.0 & 46.3 & 98.0 & 73.0 & 41.0 & 2.4 \\
\hline$C 13$ & 59.0 & 33.0 & 44.9 & 70.0 & 59.0 & 56.8 & 1.0 \\
\hline$C 15$ & -19.2 & 33.0 & -25.8 & -1.3 & -2.0 & -7.3 & 0.6 \\
\hline$C 22$ & 117.0 & 116.0 & 138.0 & 125.0 & 131.0 & 97.9 & 2.3 \\
\hline$C 23$ & 37.5 & 80.0 & 84.4 & 41.4 & 56.0 & 41.2 & 5.2 \\
\hline$C 25$ & -17.0 & 0.0 & -25.9 & -4.8 & 14.0 & -33.5 & 1.5 \\
\hline C 33 & 152.2 & 172.0 & 170.0 & 150.0 & 167.0 & 178.8 & 1.1 \\
\hline C35 & -18.7 & -16.0 & -28.8 & -16.0 & -49.0 & -5.1 & 0.9 \\
\hline$C 44$ & 27.2 & 27.0 & 24.2 & & 31.0 & 21.8 & 0.5 \\
\hline$C 46$ & 13.8 & 13.0 & 11.4 & & 10.0 & 10.6 & 0.4 \\
\hline$C 55$ & 28.2 & 36.0 & 28.4 & 28.0 & 46.0 & 19.5 & 0.6 \\
\hline$C 66$ & 39.9 & 42.0 & 31.6 & & 25.0 & 39.8 & 0.3 \\
\hline
\end{tabular}

Afanaseva et al. (Ref. 3).

${ }^{b}$ Huntington et al. (Ref. 5).

'Danno and Inokuchi (Ref. 14).

'Elnahwy et al. (Ref. 6).

e Pawley (Ref. 1).

'This work.

${ }^{g}$ Function error.

does not rely on special propagation directions, and thus the errors do not accumulate and the need for solving ambiguous quadratic or cubic equations is eliminated. Further, it removes subjective judgments, such as neglecting of certain constants which are employed in other calculations.

\section{RESULTS}

The results of the minimization method are checked for accuracy in several ways. First, a direct comparison is made between the measured velocities and the velocities calculated from the best set of elastic constant values. In this work the average error between these values was $3.3 \%$. Second, an error in the function value, Eq. (5), is obtained. This error measures how much an elastic constant value can vary before it causes a $1 \%$ change in the function, i.e., the square of the error vector. The less the value can change the better determined it is assumed to be and, thus, is similar to a standard deviation. The function is expressed in such a way that its value should be low. Therefore, the lower the function value, the better the values of the elastic constant set should be. Since this method is a least-squares procedure, increasing the data points improves the certainty in the results. In this study 58 velocities were used in the determination of the elastic constants, which is a substantial increase over the 18 generally used. In order for the elastic constant set to be real, the determinant of the $6 \times 6$ matrix must be positive. This condition was met.

The results of the elastic constant determination are given in Table I along with values from other works. These values are also compared in Fig. 3. Reasons for disagreement among the various determinations are discussed in the next section of this paper.

To clarify the role packing and molecular structure has on phonon propagation, the phonon velocity projections are plotted onto the three crystal planes. The positions of the molecules are also given (Figs. 4, 5, and 6).

\section{DISCUSSION}

\section{Present results}

As shown by Fig. 4, in the ac plane the projection of the long axis of the molecule coincides with the long axis of the longitudinal sound velocity. Similar behavior is observed in the case of polyphenyls. ${ }^{18}$ Using common arguments to obtain a basic understanding of the forces involved, one assumes that the anthracene molecule behaves as one rigid unit. This is a reasonable assumption for anthracene since the internal molecular modes are substantially higher in energy than the external lattice modes. The sound velocities in the axial directions can then be expressed as

$$
\begin{aligned}
& v_{c}=\left(k_{1} / M_{n}\right)^{1 / 2} C_{n}, \\
& v_{b}=\left(k_{2} / M_{n}\right)^{1 / 2} C_{n}, \\
& v_{a}=\left(k_{3} / M_{n}\right)^{1 / 2} C_{n},
\end{aligned}
$$

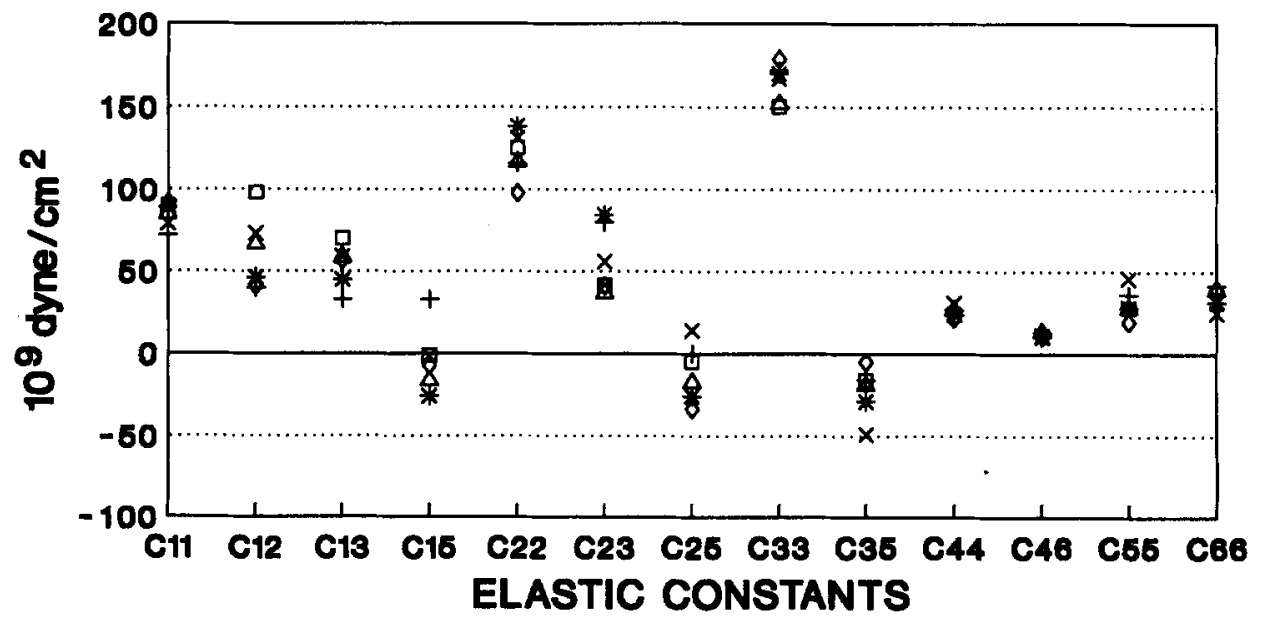

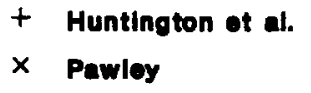

+ Huntington of al.
$\times \quad$ Pawley

J. Chem. Phys., Vol. 90, No. 4, 15 February 1989
FIG. 3. Comparison of crystalline anthracene elastic constants from various studies. 


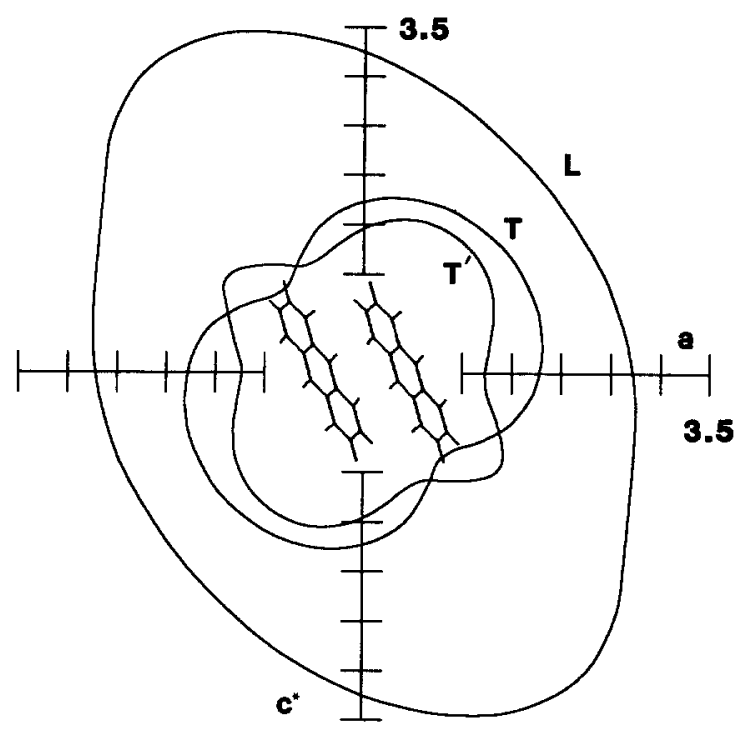

FIG. 4: Sound velocity diagram in the ac plane. Each tick mark represents $0.5 \mathrm{~km} / \mathrm{s}$. The outer curve $L$ represents the pseudolongitudinal mode velocity, whereas curve $T$ represents the pseudotransverse mode velocity and $T^{\prime}$ the out-of-plane pseudotransverse mode velocity. The interior shows the corresponding projection of the anthracene unit cell packing.

where $k_{1}, k_{2}$, and $k_{3}$ are the intermolecular "force constants" for longitudinal movement along the $\mathbf{c}, \mathbf{b}$, and a directions of the crystal; $M_{n}$ is the mass of the molecule, $C_{n}$ the distance between units, and $n$ designates the number of units.

The experimental values for $v_{c}, v_{b}$, and $v_{a}$ obtained in this study are $3.79,2.80$, and $2.72 \mathrm{~km} / \mathrm{s}$, respectively. The ratios of $k_{2} / k_{1}, k_{1} / k_{3}$, and $\mathrm{k}_{2} / \mathrm{k}_{3}$ are $1.37,1.07$, and 1.46 . These data imply that the force constant in the a direction is nearly equal to that in the c direction. The force constant in the $\mathbf{b}$ direction has to be larger than those for $\mathbf{a}$ or $\mathbf{c}$. This is consistent with the crystal's mechanical properties. The

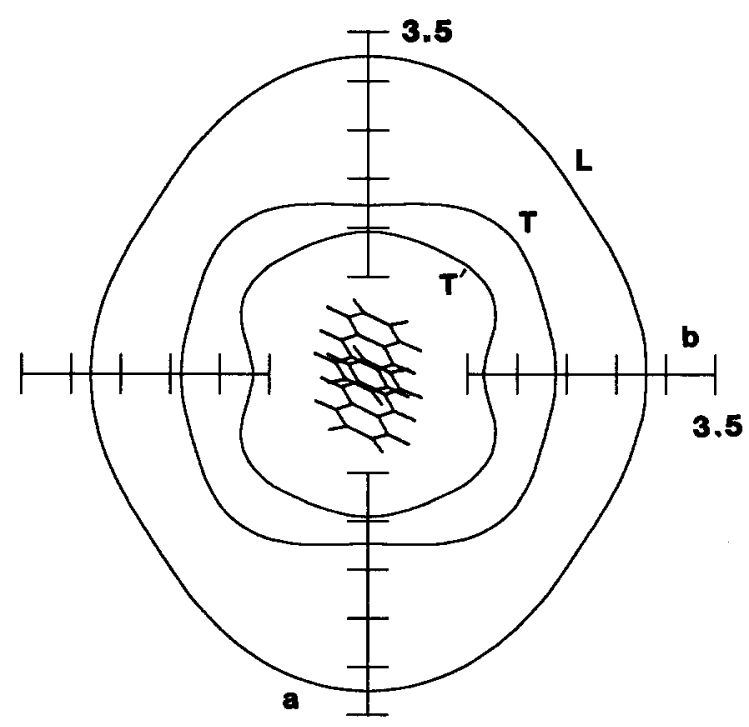

FIG. 5: Sound velocity diagram in the bc plane. Each tick mark represents $0.5 \mathrm{~km} / \mathrm{s}$. The outer curve $L$ represents the pseudolongitudinal mode velocity, whereas curve $T$ represents the pseudotransverse mode velocity and $T^{\prime}$ the out-of-plane pseudotransverse mode velocity. The interior shows the corresponding projection of the anthracene unit cell packing.

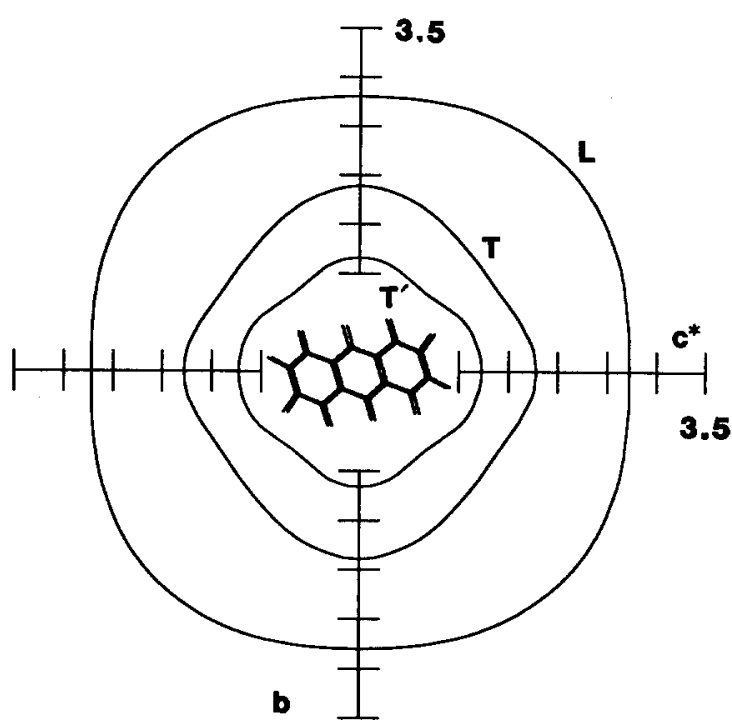

FIG. 6. Sound velocity diagram in the ab plane. Each tick mark represents $0.5 \mathrm{~km} / \mathrm{s}$. The outer curve $L$ represents the pseudolongitudinal mode velocity, whereas curve $T$ represents the pseudotransverse mode velocity and $T^{\prime}$ the out-of-plane pseudotransverse mode velocity. The interior shows the corresponding projection of the anthracene unit cell packing.

cleavage plane is perpendicular to the b axis therefore implying that the binding energy in the a or c directions is less than those along $\mathbf{b}$.

When strictly argued, this type of model does not hold since the Cauchy relations are not valid in the general case, but it is nevertheless instructive to observe behavior when they are assumed. Since under the Cauchy conditions $C_{12}$ would equal $C_{66}$, it is of interest to examine the similarities in the elastic constants. Values obtained for these in this study are nearly equal which implies that "central" forces maybe responsible for elastic interaction in these directions. ${ }^{15}$ For instance, if two opposite forces act on a center of symmetry then no internal strain will be present, i.e., the elastic constants are equal in the two opposing directions. Insightful results may also be expected when the mass is changed in a study of crystalline naphthalene. This will be a subject of future research.

\section{Comparison with previous studies}

In comparing elastic constants with other reported values one can see that agreement falls within a limited range (Table I). Taking the values as a complete set, the work by Afanaseva et al. is the best match. However, some constants in this study are in better agreement with other work. For instance, the $C_{33}$ and $C_{12}$ values of Huntington et al. are in very good agreement with the present work but disagreement is found with the sign of $C_{15}$. The derived $C_{25}$ value of Pawley also differs in sign. Other than these two exceptions, the signs of all the elastic constants coincide.

There are several possible reasons for disagreement with some of the values reported in other experimental work. In the present work, the elastic constant values are minimized to give the smallest error between the experimental phonon velocities and the phonon velocities calculated from the elas- 
tic constants. For better values represented by a deeper minimum in the error vector, over three times the usual number of velocities are utilized. This gives a better statistical sampling for calculation of the elastic constants. Also, by minimizing, the error in calculating the values does not accumulate. In other calculation methods, errors can accumulate differently. For instance, since the $C_{46}$ element is related to the (101) wave-vector direction by

$$
\rho v^{2}=\frac{1}{2}\left(C_{44}+C_{66}\right)+C_{46}
$$

and the $(010)$ wave-vector direction by

$$
\rho v^{2}=\frac{1}{2}\left\{\left(C_{44}+C_{66}\right) \pm\left[\left(C_{44}-C_{66}\right)^{2}+4 C_{46}\right]^{1 / 2}\right\}
$$

it is possible to obtain two different values depending on which wave-vector direction is chosen for the calculation. The two values would express the different errors associated with the two paths of calculation. The off-diagonal elements of the elastic constant tensor are generally the values with the largest accumulated error. Since the present method avoids accumulating errors, this can cause some values of other studies to agree with the present work while disagreeing with other values.

The optical and mechanical quality of the crystal is another important consideration. The experimental method employed in this work allows the crystal to remain uncut which preserves its integrity. Finally, the crystal used in this study was vapor grown. Vapor grown molecular crystals have much fewer internal strains than those that are melt grown. Highly strained crystals or those damaged by cutting may be expected to give a much different set of elastic constants and admit greater variability in their values. A lower defect concentration solid is softer in most directions and the general trend of values for the elastic constants reported here are consistent with this when compared to those previously reported.

Of greater interest is the comparison of experimental to the theoretical values because these differences show how well the system is understood. If one assumes the 6-exp potential function used by Pawley accurately describes this system, then reasons for these differences can be proposed. A major cause for them is that the experimental system is a dynamic system, whereas the calculated system is assumed static. This can affect the reported values.

static. This can affect the reported values.

When an experimental elastic constant is lower than its theoretical value one infers that the phonon may be coupled to a lattice or molecular mode which dampens its velocity. ${ }^{19}$ For instance, the $C_{22}$ constant has a much smaller experimental than theoretical value. This constant is associated with the b direction. From Figs. 5 and 6 one can see that the phonon velocities propagating in this direction are relatively slow. This may be reasonably attributed to the phonon coupling to the molecular libration about its long axis, since this libration is of low energy and proper symmetry for coupling. The quasitransverse mode in the scattering plane of Fig. 4 shows reduced velocities in the $\mathrm{c}$ direction. This is consistent with the rotational coupling in the b direction which is of interest, because this type of coupling is responsible for the dissipation of heat by crystals. It may be shown by studies such as this one, that crystals dissipate heat in a very selective fashion. ${ }^{19}$

On the other hand, the $C_{11}$ value is higher than the theoretical value suggesting that the van der Waals interaction in the a direction is larger than expected for this type of interaction. From Fig. 6 it is apparent that the end hydrogens, i.e., those in the $2,3,6$, and 7 positions of the molecule, may have a rather large interaction. This end-to-end hydrogen interaction is again seen in the c direction (Fig. 4) and also results in a large elastic constant value $C_{33}$ for this direction. A possible reason for this consistent disagreement is that the end hydrogens may have slightly longer bond lengths than the $\mathrm{x}$ ray data show. It is generally accepted that the exact location of hydrogens is difficult to determine. Another consideration is that in the state of dynamic motion the average mass in this direction is greater than previously realized.

\section{CONCLUSION}

This work reports the measured sound velocities in numerous directions of crystalline anthracene by Brillouin scattering. From these, a complete set of elastic constants are calculated. Straightforward arguments are used to gain some insight into the relation of the phonon propagation and the molecular packing.

Also, it is shown that the technique of minimizing a calculated set of velocities to an experimental set in order to obtain elastic constants is an effective and accurate method, even for low symmetry crystals. This approach is completely general and does not rely on special wave-vector directions in the crystal. Using this minimization method also circumvents the accumulation of errors inherent in other approaches. One of its major advantages is that the crystal can remain uncut. This avoids depending on the precision of the cuts of crystals as well as averting the introduction of defects. Also, since cutting is not necessary, much smaller crystals can be used which are, in turn, apt to be more defect-free.

With improved experimental and calculational methods employed in this study, advancement in the theoretical calculations will be possible. This may be expected to lead to further refinement of theoretical lattice dynamical calculations. For instance, the intermolecular interaction of end-toend hydrogens may be quite different from hydrogens that are above and below one another even though their distances may be the same. Progress may be made by not only considering atom-atom distances but also by including atom-atom angles. Future calculative approaches may contain orientational fluctuations or damping constants.

\section{ACKNOWLEDGMENTS}

Support for this research by a grant from 3M Corporation is greatly appreciated. We thank Professor Norbert Karl for providing the crystals. We are also grateful to Dr. D. P. Billesbach and Dr. K.-H. Brose for their help.

'G. S. Pawley, Phys. Status Solidi 20, 247 (1967).

${ }^{2}$ A. I. Kitaigorodskii, J. Chem. Phys. 63, 1 (1966). 
${ }^{3}$ G. K. Afanaseva, K. S. Aleksandrov, and A. J. Kitaigorodskii, Phys. Status Solidi 24, 61 (1967).

${ }^{4}$ T. Danno and H. Inokuchi, Bull. Chem. Soc. Jpn. 41, 1783 (1968).

${ }^{5}$ H. B. Huntington, S. G. Gangoli, and J. L. Mills, J. Chem. Phys. 50, 3844 (1969).

'S. Elnahwy, M. El Hamamsy, A. C. Damask, D. E. Cox, and W. B. Daniels, J. Chem. Phys. 68, 1161 (1978).

${ }^{7}$ W. Hayes and R. Loudon, Scattering Of Light By Crystals (Wiley, New York, 1978).

${ }^{8}$ R. Mason, Acta Crystallogr. 17, 547 (1964).

${ }^{9}$ A. N. Winchell, The Optical Properties Of Organic Compounds (Academic, New York, 1954).

${ }^{10}$ I. L. Fabelinskii, Molecular Scattering of Light (Plenum, New York, 1968).
'S. M. Shapiro, R. W. Gammon, and H. Z. Cummins, Appl. Phys. Lett. 9, 157 (1966).

${ }^{12}$ H. Z. Cummins and R. W. Gammon, Appl. Phys. Lett. 6, 171 (1965).

${ }^{13}$ H. Z. Cummins and A. P. Levanyuk, Light Scattering Near Phase Transitions (North-Holland, Amsterdam, 1983).

${ }^{14}$ M. C. Tobin, Laser Raman Spectroscopy (Wiley, New York, 1971).

${ }^{15} \mathrm{G}$. Venkataraman, L. A. Feldkamp, and Y. C. Sahni, Dynamics of Perfect Crystals (MIT, Cambridge, 1975).

${ }^{16}$ S. F. Ahmad, H. Kiefte, M. J. Coulter, and M. D. Whitmore, Phys. Rev. B 26, 4239 (1982).

${ }^{17}$ K. H. Brose and C. J. Eckhardt, Chem. Phys. Lett. 125, 235 (1986).

${ }^{18} \mathrm{C}$. Ecolivet and M. Sanquer, J. Chem. Phys. 72, 4145 (1980).

${ }^{19}$ L. N. Liebermann, in Physical Acoustics, edited by W. P. Mason (Academic, New York, 1966), Vol. 4, p. 183. 